\title{
Quantifying the Interaction Stages of a Public Display Campaign in the Wild
}

\author{
Gonzalo Parra, Robin De Croon, Joris Klerkx, and Erik Duval \\ Dept. of Computer Science, University of Leuven \\ Celestijnenlaan 200A B-3001 Leuven, BE \\ \{gonzalo.parra, robin.decroon, joris.klerkx, erik.duval\}@cs.kuleuven.be
}

\begin{abstract}
In this paper we present the findings from three exploratory studies in the wild of an interactive public display aiming to increase awareness on cardiac arrest and Cardiopulmonary Resuscitation (CPR). Three different locations (train station, hospital, and university restaurant) were selected in order to understand how context affects the effectiveness of the reallife campaign. For this purpose, we defined and quantified different interaction phases based on the audience funnel and the characteristics of the prototype. Our results confirm that context (location and people) have a direct effect on engagement throughout the interaction phases. A location that clearly relates to the content of a campaign or has an audience that is able and willing to interact will positively influence the outcome of a campaign. In addition, we show that following a model to quantify and compare studies is a feasible and useful approach.
\end{abstract}

\section{Author Keywords}

Interactive public displays; audience funnel; interaction stages; context.

\section{ACM Classification Keywords}

H.5.1. Multimedia Information Systems: Evaluation/methodology

\section{INTRODUCTION AND BACKGROUND}

Urban public spaces are being increasingly populated by large displays and are being used for different purposes, from marketing to information services. Currently, these public displays are becoming interactive and context-aware. To reach a better understanding of this technology, several studies in the wild evaluate and present how passers-by react, interact, and engage with public displays [1, 5, 8]; but most of them do not use proposed models (such as the "audience funnel" [4]) to enable comparison and benchmark of results between them, or with future research work. In this paper, and based on the audience funnel, we quantify and report the results of three exploratory studies in the wild of the same interactive public display aiming to comprehend how context

Permission to make digital or hard copies of all or part of this work for personal or classroom use is granted without fee provided that copies are not made or distributed for profit or commercial advantage and that copies bear this notice and the full citation on the first page. Copyrights for components of this work owned by others than the author(s) must be honored. Abstracting with credit is permitted. To copy otherwise, or republish, to post on servers or to redistribute to lists, requires prior specific permission and/or a fee. Request permissions from Permissions@ acm.org.

NordiCHI '14, October 26 - 30 2014, Helsinki, Finland

Copyright is held by the owner/author(s). Publication rights licensed to ACM. ACM 978-1-4503-2542-4/14/10\$15.00.

http://dx.doi.org/10.1145/2639189.2639216 affects the different interactive stages of passers-by. Particularly, we want to observe and understand how an interactive prototype dealing with an important subject, such as cardiac arrest and Cardiopulmonary Resuscitation (CPR) awareness, behaves in different contexts.

In respect to context, and based on the concept that any piece of information that characterizes the situation of a participant in an interaction is considered to be part of it [3]; there are various studies that have evaluated context using different information elements, such as location and people. For example, [8] focused on three elements: (i) the space (as the geometric structure including visual and auditory displays), (ii) the people currently inhabiting it (including the active users) and (iii) the rules implied by society as social rules and the rules in the context of the individual person. This study found that immediacy invites people to just give it a try, without the need to wait in a queue. It is the people and space that need to be taken into account for catching the people's attention, but it is mostly the rules that result in motivation.

Interactive displays often fail to deal appropriately with the social inhibitions associated with interaction in public. Initial engagement is challenging as people generally expect displays to show irrelevant content, such as advertising. This phenomenon is referred as "display blindness" [6]. In order to increase engagement rates, [1] analyzed the effects of supporting space or social context (also referred as place). From different context studies, place trumps space [1]: a conducive social context could overcome a poor physical space and encourage interaction. Conversely, an inappropriate social context could inhibit interaction in spaces that might normally facilitate engagement. During our evaluation studies, we also found indications that confirm this statement. The social context of a challenging space can be manipulated to encourage engagement, by using comperes for example [1]. Yet, it is difficult to work against existing social constraints, irrespective of the facilitation of spatial factors.

In [9], a conceptual framework is presented in order to position situated engagement across three key parameters: people, content, and location. As a result, a method to maximize engagement by identifying a "sweet spot" within the intersection of these parameters was proposed. This sweet spot is reached when you have (i) the right location for your display (including size, orientation, and screen positioning), (ii) have a young, tech savvy, and homogeneous audience; and (iii) the right content that they are passionate about. From 

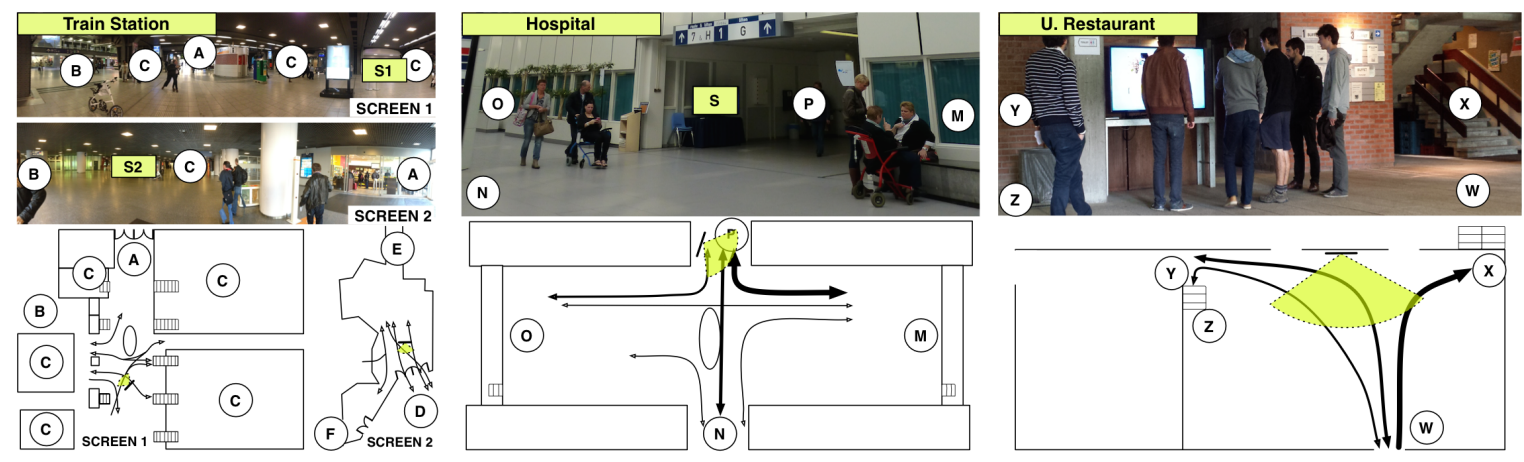

Figure 1. Location of the screens in the train station (left), hospital (middle), and university restaurant (right).

their results, this sweet spot minimizes the social inhibitions of public input methods [2].

For the purpose of our studies, we vary on two defining elements of context, location and people, and investigate its influence on the engagement and effectiveness of a real-life campaign. To achieve this, we evaluated one interactive prototype in three different contexts: (i) international train station (general location with busy public), (ii) hospital (location related to the campaign content), and (iii) university restaurant (open and tech savvy public). With these locations we also aim to evaluate the sweet spot concept to increase engagement [9]. Particularly, we expect that the hospital context be the closest to the sweet spot. Finally, we report and discuss on the results and observations using the audience funnel as a model to focus on each of the interactive stages.

\section{STUDIES}

For our studies, an interactive prototype from our previous work is used [7]. This prototype is designed to raise consciousness regarding actions laymen can take when somebody has a cardiac arrest. The different phases of this prototype are depicted in Figure 2. Each time a passer-by is detected the screen presents a user representation (step 1). We used two variations of the representation (silhouette and mirror), based on the outcome of previous work of Müller et al. [5]. The variants were rotated in order to reduce the effects of the deployment hour in the results. Then, the prototype determines the skeleton of the user and overlays a beating heart over the chest of the user (step 2). This step tries to use the effect of immediacy to capture people to try the system [8]. After five seconds of heart beating, it "stops" (simulating a problem with the user's heart) and the call-to-action text "Touch your heart!" is shown (step 3). If the user perform this gesture the final screen of the prototype is reached: a twenty second video on cardiac arrest awareness together with an invitation message to take a flyer that lies next to the screen (step 4). This flyer contains more information about
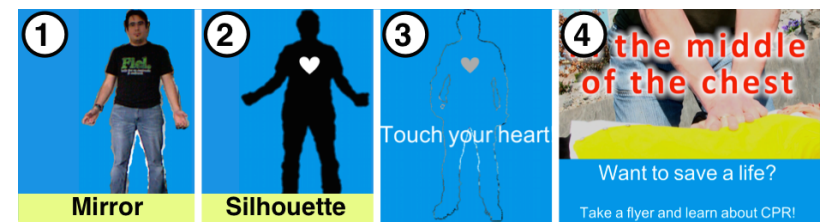

Figure 2. Phases of the prototype [7]. the campaign and a link to its website. Interactions took from 6 seconds (reaching step 4) to 30 seconds (watch full video).

For the deployment, 60" and 65" LCD TVs in landscape mode, a Microsoft Kinect camera, and a laptop for real-time computations were used. The software to render the display builds on Processing and uses the OpenNI framework to detect the passers-by and their interactions. The different contexts of our studies were selected based on two of the "sweet spot" elements [9]: location and people; while the content element was fixed. Screen locations in our different contexts were selected on the basis of: (i) having a large number of passers-by, (ii) avoiding disturbance of normal walking flows, (iii) orienting the screens to capture natural passers-by flows, (iv) considering security regulations of the location, and (v) minimize potential technical limitations of the system. Taking these into account, we tried to get close to the sweet spot as much as possible.

For our evaluation, we collected quantitative data from the interaction logs of the prototype and images captured by the Kinect camera. For this purpose, we apply state-of-art computer vision algorithms to detect and generate the passer-by count. Also, researchers were strategically located in order to observe and take notes regarding the behavior of the passersby without being seen. The logs were also used to contrast and corroborate the annotations made.

\section{Train Station}

The public display was deployed during one day in two different locations of the biggest railway station of Brussels, Belgium. Both screens were running concurrently but were located in opposite entrances of the station to avoid people potentially passing by the two screens. The location of the displays and the flows of passers-by are depicted in Figure 1. Screen 1 was located in the main hall of the station, where it was exposed to different flows of passers-by such as: flow from the entrance to the station (A), arrivals from the different underground metro lines (B), and access to, from, and between the different train tracks (C). Screen 2 was located in a secondary hall of the train station, which had fewer passerby flows and was exposed to a smaller number of potential users. These flows were from the entrance (D) to the hall that provides access to the train tracks (E) and from a connecting hall $(\mathrm{F})$ between the main and the secondary halls.

\section{Hospital}




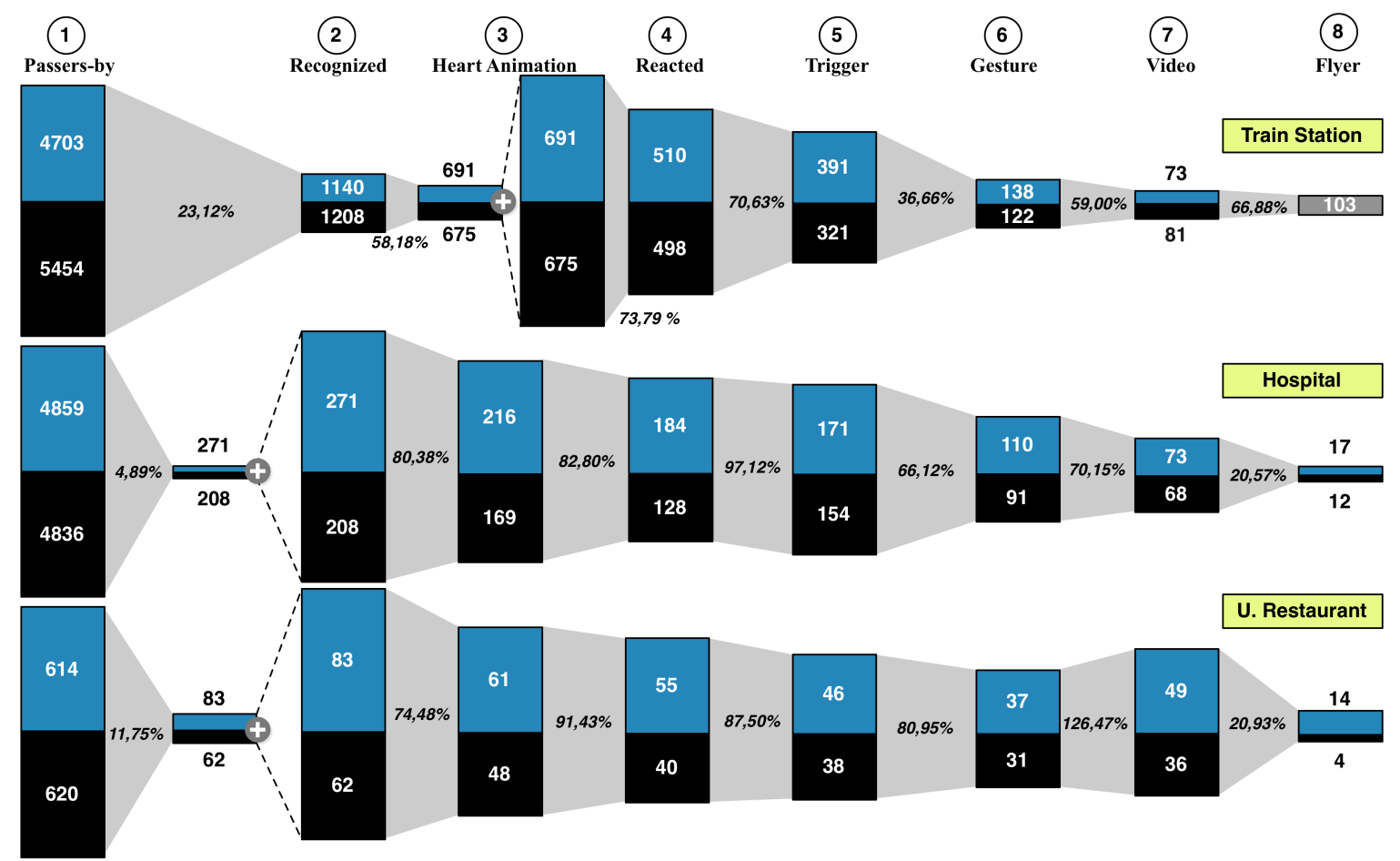

Figure 3. Flow stages (pass-by, user recognition, overlay heart animation, reaction, trigger to gesture, perform gesture, observed video, and flyer) from the three studies. The blue sections represent the results obtained by the mirror representation, while the black ones represent the silhouette.

In a second study, we deployed the public display for two days at the Academic Hospital of Maastricht (azM), in The Netherlands. This is the main hospital of the city with around 444.000 yearly visits and 5204 employees. The location of the display and the flow of passers-by are depicted in Figure 1. The display was installed in the main entrance and was exposed to three main flows of passers-by coming from the parking of the hospital (M), public transportation: buses and taxis $(\mathrm{N})$, and a train station $(\mathrm{O})$. The highest flow was from the parking to the reception zone $(\mathrm{P})$.

\section{University Restaurant}

The public display was deployed for a day during lunchtime in the second biggest university restaurant of KU Leuven, Belgium. This is the main restaurant in the science and engineering faculty with around 1000 visits during lunchtime. The location of the display and the flow of passers-by are depicted in Figure 1. The screen was exposed to three main flows, one from the main entrance (W) to the stairs leading to the restaurant $(\mathrm{X})$, another from the entrance to a sandwich bar $(Y)$, and one from the exit stairs $(\mathrm{Z})$ to the entrance $(\mathrm{W})$.

\section{RESULTS AND DISCUSSION}

In order to have a more detailed analysis of results we defined and quantified eight stages of flow: (1) pass-by the display (with or without noticing it), (2) user detected by the system (getting user representation), (3) beating heart animation, (4) reaction, (5) trigger for gesture (stopped heart), (6) performing gesture (touching heart), (7) observe the full video, and (8) take a campaign flyer. These were chosen considering the interactive stages from the audience funnel [4] together with the design characteristics of the prototype. We grouped the initial stages of the audience funnel due to technical limitations. Also, we added specific prototype stages to quantify the attracting cues (user representation and heart animations) and the follow-up actions (video and flyers). The Sankey diagrams in Figure 3 visualize the results of the different stages from the three studies. The percentages are calculated based on the previous step. We use two user representations, silhouette and mirror, in order to compare their effectiveness. The blue sections of the different stages represent the results obtained by the mirror representation, while the black ones represent the silhouette results.

As reported before [7], in the three studies most of the passers-by did not stay long enough to get a heart animation or even to be recognized by the system (stage 1 to 2 ). This situation is more visible in the hospital and the university restaurant $(4,89 \%$ and $11,75 \%$ respectively). These results can be partially justified by the locations of the screens. While those aimed to maximize the number of passers-by, these were not always the best spots due to the constraints explained in the previous section. Moreover, the hospital and restaurant were exposed to more parallel screen flows than frontal, making the noticing technique less effective. Despite these issues, and as previously observed $[5,7]$, a live user representation shows to be an effective way to capture people's attention. Following the stages, the restaurant and hospital obtained a higher percentage from recognized users that got a heart representation ( 2 to 3 ), these are explained by the walking speed of the passers-by in these context. In the train station, the people were in a hurry to catch a train or a metro. Considering the reaction to the heart animation (3 to 4), the university restaurant got the highest percentage $(91,43 \%)$ due to the type of 
audience. Indeed, visitors (mostly in groups) were prone to play and have fun in front of the display but were less interested in the content of the campaign as in some cases they immediately left after seeing the video and the final text. In the hospital study, patients were focused on finishing their tasks (e.g.: being on time for doctor's appointment), but were more relaxed and prone to interact with the display when leaving the hospital. This behavior was also observed, in less quantity, in the restaurant.

The train station was the least effective into capturing people to stay until the heart is stopped (stage 4 to 5). In some cases, these percentages were affected by users that were figuring out which gesture to use, or touched the display instead of the heart representation. This is applicable, even more strongly (only $36,66 \%$ ), to performing the gesture (5 to 6). From these we can conclude that our public display using gesture based interaction (e.g.: touching your heart) does not performs as expected in places were people have less time or interest. As happens in our case, due to the transient nature of the train station while these displays are more focused in being playful and performative. Towards the final stages of the prototype, the passers-by from the university restaurant got the highest percentage of watching the video $(126,47 \%$ from stage 6 to 7). This percentage increased dramatically as the audiences were in most cases in groups, which increased the number of persons in the video stage. Regarding the last stage (taking the flyer, 7 to 8), while users from the hospital and university seem more receptive to this mean of communication we observed that the train station got the highest percentage; as they are probably used (or are interested) to get information in this way (they took flyers even without interacting with the display). In a broader view, people from the train station who trigger the video tend to also continue toward the later stage, which is not the case in the other contexts.

In the prototype, the length of the attracting, arriving, and interacting sequence or arriving in the middle of the interaction was a problem that got magnified due to the nature of the context. On the other hand, novelty is still a big factor affecting positively to engagement. In both the hospital and restaurant, passers-by were prone to try the system and play with it; particularly in the restaurant, where passers-by had more spare time. Regarding attracting and engaging phenomena observed in previous studies, the honey-pot [2] and landing effects [5] are common in settings were passers-by tend to move in groups. In our case, the restaurant was the ideal context to observe this phenomenon. Also, the sweet spot (people, location, content [9]) was assumed to be present in the hospital study due to the content of the campaign (cardiac arrest and CPR awareness), but it had no big effect in our case. To conclude, and as seen in Figure 3, we did not observe that the mirrored representation of the passer-by clearly outperforms the silhouette representation; as reported in previous studies [5].

\section{CONCLUSIONS}

In this paper we quantified interaction stages of a public display campaign in three different contexts in order to analyze the effect of it. From our results we can confirm that the con- text of the deployment location can hinder the engagement of the prototype. For example, a busy context such as the train station has overall lower retention percentages throughout the interaction phases. Furthermore, our public display using gesture based interaction does not perform as expected in places where people have less time or interest. On the other hand, a location that clearly relates to the content of the campaign (in our case the hospital) or has an audience with the availability and more open to interact (university restaurant), positively affects engagement.

Effects reported by other studies, such as the honey-pot [2] and landing effects [5], are common in contexts where passers-by move in groups. Finally, we conclude that despite the difference in context, a live user representation is an effective way to capture people's attention. Yet in our studies, we were not able to confirm that a mirrored image of a user clearly outperforms the use of a silhouette.

\section{ACKNOWLEDGMENTS}

We gratefully acknowledge the support of the European Regional Development Fund (ERDF), several regions of the Euregio Meuse-Rhine, and the EMuRgency project partners.

\section{REFERENCES}

1. Akpan, I., Marshall, P., Bird, J., and Harrison, D. Exploring the effects of space and place on engagement with an interactive installation. In Proc. $\mathrm{CHI}$ ' $13, \mathrm{ACM}$ (2013), 2213-2222.

2. Brignull, H., and Rogers, Y. Enticing people to interact with large public displays in public spaces. In Proc. INTERACT 2003, IOS Press (2003), 17-24.

3. Dey, A. K., and Abowd, G. D. Towards a better understanding of context and context-awareness. In Proc. HUC 99, Springer-Verlag (1999), 304-307.

4. Müller, J., Alt, F., Michelis, D., and Schmidt, A. Requirements and design space for interactive public displays. In Proc. MM '10, ACM (2010), 1285-1294.

5. Müller, J., Walter, R., Bailly, G., Nischt, M., and Alt, F. Looking glass: a field study on noticing interactivity of a shop window. In Proc. CHI '12, ACM (2012), 297-306.

6. Müller, J., Wilmsmann, D., Exeler, J., Buzeck, M., Schmidt, A., Jay, T., and Krüger, A. Display blindness: The effect of expectations on attention towards digital signage. In Pervasive Computing, vol. 5538 of Lecture Notes in Computer Science, Springer-Verlag (2009), 1-8.

7. Parra, G., Klerkx, J., and Duval, E. Understanding engagement with interactive public displays: An awareness campaign in the wild. In Proc. PerDis '14, ACM (2014), 180-185.

8. Praxmarer, R., and Wagner, T. Playfulness and large-scale interactive projections in public space. International SERIES on Information Systems and Management in Creative eMedia 0, 3 (2013), 18-30.

9. Schroeter, R., Foth, M., and Satchell, C. People, content, location: Sweet spotting urban screens for situated engagement. In Proc. DIS '12, ACM (2012), 146-155. 